\title{
The haplotype-based analysis of Aegilops tauschii introgression into hard red winter wheat and its impact on productivity traits
}

\author{
$1 \quad$ Moses Nyine', Elina Adhikari' ${ }^{1}$, Marshall Clinesmith ${ }^{2}$, Robert Aiken², Bliss Betzen ${ }^{1}$, Wei \\ 2 Wang ${ }^{1}$, Dwight Davidson ${ }^{1}$, Zitong Y $\mathbf{u}^{1}$, Yuanwen Guo ${ }^{1}$, Fei He ${ }^{1}$, Alina Akhunova ${ }^{3}$, Katherine \\ 3 W Jordan ${ }^{1,4}$, Allan K Fritz ${ }^{2}$, Eduard Akhunov ${ }^{1 *}$. \\ 4 \\ 5 \\ 6 \\ ${ }^{1}$ Department of Plant Pathology, Kansas State University, Manhattan, USA \\ ${ }^{2}$ Department of Agronomy, Kansas State University, Manhattan, USA \\ ${ }^{3}$ Integrated Genomics Facility, Kansas State University, Manhattan, USA \\ ${ }^{4}$ USDA-ARS Hard Winter Wheat Genetics Research Unit, Manhattan, USA \\ *Corresponding author: \\ Eduard Akhunov \\ Email: eakhunov@ksu.edu
}

Keywords: wheat, Aegilops tauschii, wild relative introgression, whole genome sequencing, haplotypes, genotype imputation, grain yield, yield component traits.

\begin{abstract}
Introgression from wild relatives have a great potential to broaden beneficial allelic diversity available for crop improvement in breeding programs. Here, we assessed the impact of introgression from 21 diverse accessions of Aegilops tauschii, the diploid ancestor of the wheat $\mathrm{D}$ genome, into six hard red winter wheat cultivars on yield and yield component traits. We used 5.2 million imputed D genome SNPs identified by whole-genome sequencing of parental lines and the sequence-based genotyping of introgression population including $351 \mathrm{BC}_{1} \mathrm{~F}_{3: 5}$ lines. Phenotyping data collected from the irrigated and non-irrigated field trials revealed that up to $23 \%$ of the introgression lines produce more grain than the parents and check cultivars. Based on sixteen yield stability statistics, the yield of twelve introgression lines (3.4\%) was stable across treatments, years and locations; five of these lines were also high yielding, producing $9.8 \%$ more grain than the average yield of check cultivars. The most significant SNP-trait and haplotype-trait associations were identified on chromosome arms 2DS and 6DL for spikelet number per spike (SNS), on chromosome arms 2DS, 3DS, 5DS and 7DS for grain length and on chromosome arms 1DL, 2DS, 6DL and 7DS for grain width. Introgression of haplotypes from Ae. tauschii parents was associated with increase in SNS, which positively correlated with heading date, whereas haplotypes from hexaploid wheat parents were associated with increased grain width. We show that haplotypes on 2DS associated with increased spikelet number and heading date are linked with multiple introgressed alleles of $P p d-D 1$ identified by the wholegenome sequencing of the Ae. tauschii parents. While some introgressed haplotypes exhibited significant pleiotropic effects with the direction of effects on the yield component traits being largely consistent with the previously reported trade-offs, there were haplotype combinations associated with the positive trends in yield. The characterized repertoire of the introgressed haplotypes derived from Ae. tauschii accessions with the combined positive effects on yield and yield components traits in
\end{abstract}


elite germplasm provides a valuable source of alleles for improving the productivity of winter wheat by optimizing the contribution of component traits to yield.

\section{Number of words: 8442 , Number of figures: 5}

\section{$42 \quad 1 \quad$ Introduction}

The gap between population expansion and food production is increasing due to marginal improvements in crop yield, which is attributed to declining soil fertility, pests and diseases, and climate change (Bailey-Serres et al., 2019). Wild relatives of wheat are a rich source of novel underutilized allelic diversity with great potential to improve cultivated wheat through introgression (Placido et al., 2013; Zhang et al., 2017; Hao et al., 2020). Introgression from wild relatives into elite wheat cultivars was reported to increase pest and disease resistance (Periyannan et al., 2013; Saintenic et al., 2013), improve resilience towards environmental stress (Peleg et al., 2005; Placido et al., 2013) and increase yield (Pasquariello et al., 2019). The success of introgression breeding, however, could be affected by the negative epistasis between the multiple alleles of wild and cultivated wheat (Nyine et al., 2020), especially in the low recombing regions of chromosomes, where linkage with the negatively selected alleles could reduce the efficiency of selection for beneficial variants (Hill and Robertson 1966).

Introgression could exhibit pleotropic effects, affecting multiple, often unrelated traits not directly targeted by selection. For example, introgression from Ae. ventricosa into chromosome 7D of wheat was associated with increase in grain protein content and resistance to eyespot at the expense of reduced yield (Pasquariello et al., 2019). In durum wheat, introgression of the GNI-AI gene from wild emmer increased grain weight by suppressing the fertility of distal florets, resulting in a negative correlation between grain number and grain weight (Golan et al., 2019). Introgression from Agropyron elongatum into the 7DL chromosome arm of wheat that is known to confer leaf rust resistance (Lr19) (Wang and Zhang 1996) also influences root development, resulting in improved adaption to water stress (Placido et al., 2013) and salinity (Dvorak et al., 1988), and increased biomass and yield (Reynold et al., 2001).

Crop yield is a complex trait determined by many component traits, such as thousand gain weight, grain number per spike, spikes per unit area, grain width, area and length among others (Del Moral et al., 2003; Du et al., 2020; Zhang et al., 2018). Previous studies have shown that introgression from wild and close relatives improve yield of hexaploid wheat by changing yield component traits through the pleotropic interaction between introgressed and background alleles of the hexaploid wheat (Jones et al., 2020). Significant trade-offs between yield, yield components and yield stability have been reported in wheat. A study by Pennacchi et al. (2019) showed that yield and yield stability have a negative linear relationship in most cases. Other factors such as heading date, plant height and biomass influence the source-sink ratio, which in turn affects the harvest index leading to variation in yield and yield stability (Reynold et al., 2001; Reynold et al., 2020). Balancing the trade-off between yield components is therefore necessary to improve yield, maximize the yield potential and improve yield stability in wheat.

Sequence-based genotyping generates high-density SNP marker data that could be used to accurately detect the boundaries of genomic segments introgressed from wild relatives (Kuzay et al., 2019; Nyine et al., 2020), providing a unique opportunity to investigate the effect of introgression on trade-off between traits affecting total yield. Even though whole genome sequencing became less expensive, it is still not within the cost range that would allow wheat breeding programs to sequence 
large populations. Sequencing of founder lines at a high coverage depth and using these genotypes as a reference panel to impute missing and ungenotyped markers in the progeny characterized by lowcoverage SKIM sequencing is a cost-effective alternative. The existing imputation algorithms (Browning and Browning 2013; Swarts et al., 2014; Davies et al., 2016) provide highly accurate whole-genome prediction of missing genotypes, and were shown to increase the power of genomewide association scans, thus enabling the identification of SNPs or haplotypes associated with the traits of interest (Li et al., 2010; Nyine et al., 2019). One of the advantages of the increased marker density provided by whole-genome sequencing is the ability to perform association mapping using haplotype information, which improves the detection of quantitative trait loci that would otherwise be missed when using single SNPs (Zhang et al., 2002; Lorenz et al., 2010).

Here we investigated the impact of introgression from Ae. tauschii into hard red winter wheat lines on yield and yield component traits and how haplotypes from Ae. tauschii at different genomic loci affect the component traits and total yield. For this purpose, we assessed the phenotypic variability of yield and the component traits, biomass and tenacious glume traits in an introgression population derived from Ae. tauschii and hexaploid winter wheat phenotyped under irrigated and rainfed conditions. We applied SNP diversity data generated by the whole genome shotgun sequencing at 10x coverage level of the parental lines to impute genotypes in this population (Nyine et al., 2020). This strategy resulted in 5.2 million SNPs that enabled us to identify the introgressed Ae. tauschii haplotypes and assess their effects on trait variation through genome-wide association mapping and haplotype block effect analysis. This introgression population along with high-density SNP genotype data provides a valuable resource for effective deployment of Ae. tauschii haplotypes in winter wheat improvement programs.

\section{$2 \quad$ Materials and Methods}

\subsection{Plant materials}

The study population was described in detail by Nyine et al. (2020). In brief, the population was developed by crossing synthetic Ae. tauschii-wheat octoploid lines with recurrent hexaploid winter wheat lines. The octoploid lines were developed by crossing five hexaploid winter wheat lines with 21 diverse Ae. tauschii accessions. The resulting $\mathrm{F}_{1}$ hybrid plants regenerated from rescued embryos were treated with colchicine to produce the synthetic octoploids (Dale et al., 2017). The synthetic octoploids were backcrossed once to the respective hexaploid wheat parents or to another wheat line. The $\mathrm{BC}_{1} \mathrm{~F}_{1}$ plants (hexaploid) were self-pollinated and advanced by single seed descent to the $\mathrm{BC}_{1} \mathrm{~F}_{3}$ generation. Seeds from individual $\mathrm{BC}_{1} \mathrm{~F}_{3}$ plants were bulked and grown in single rows in the field at the Kansas State University, Ashland Research Farm near Manhattan, KS in the 2016-17 growing season. A total of 351 lines were selected from the entire population based on the ability to produce sufficient seeds to allow for yield testing, general fitness, threshability and phenology corresponding to the adapted wheat cultivars.

\subsection{Field phenotyping}

The population was phenotyped in 2018 and 2019 at Colby (KS), and in 2020 at Ashland (KS). In both locations and years, an augmented design was used to establish the trials. Plots were planted using a New Holland 6-row wheat drill. Plot dimensions were 2.5 meters long by 0.5 meters wide with $18 \mathrm{~cm}$ row spacing. Starter fertilizer was applied with the seed at planting using granular 18-46-0 diammonium phosphate (DAP) at a rate of $168.1 \mathrm{~kg} / \mathrm{ha}$. Additional nitrogen was applied as a topdress in the spring using liquid 28-0-0 urea ammonium nitrate (UAN) at a rate of $67.3 \mathrm{~kg} / \mathrm{ha}$. A lateral irrigation system was used at Colby to ensure uniform germination and emergence as well as 
to provide additional water throughout the growing season in the irrigated treatment. Three hexaploid winter wheat lines well-adapted to Kansas environments (checks) and the hexaploid wheat parents were used as controls with at least three biological replications per block. In 2018 and 2019, two complete blocks were established and one block was irrigated (COI18 and COI19, respectively) whereas the other was rainfed/non-irrigated (CO18 and CO19, respectively), simulating an optimal and farmer-field growth conditions. In 2020, only one block was grown under rainfed conditions (AS20).

The population was phenotyped for yield and yield components traits, biomass traits and tenacious glume. Agrobase software (Mulitze 1990) was used to adjust the grain yield (GY), (bushels per acre) for spatial variability. The MARVIN seed imaging system (GTA Sensorik GmbH, Germany) was used to assess the grain morphometric traits such as grain number (GN) per sample, thousand grain weight (TGW), grain area (GA), grain width (GW) and grain length (GL) from two technical replicates in 2018, and one measurement in 2019 and 2020. In 2019 and 2020, data were collected for the number of spikes per square foot (SPSF) from two random points within a plot. The $1 \mathrm{ft} \times 1 \mathrm{ft}$ square frame was dropped over two rows at least one foot away from the plot edges to avoid the border effect. In 2019, only one row within a frame was cut above the ground level for biomass determination, while in 2020 , both rows were sampled. Biomass samples were collected in paper bags and dried for at least three weeks at $32^{\circ} \mathrm{C}\left(90^{\circ} \mathrm{F}\right)$ before processing. We collected data on aboveground dry biomass (BM) measured as the total weight of the dry sample without the bag, the number of spikes per sample (SPB), the average spikelet number per spike (SNS) from 10 random spikes and grain weight after threshing (GSW). During threshing, we scored samples for presence and absence of the tenacious glume (Tg) trait depending on the threshability. Harvesting index (HI) was calculated as the percentage of GSW relative to BM.

In 2020, data for heading date (HD) were collected from each plot when approximately $50 \%$ of the spikes had emerged from the flag leaves. The number of days to heading were calculated as the difference between the heading and planting dates. After all the plots had completed heading, plant height $(\mathrm{PH})$, in centimeters was measured on the same day from two random but representative main tillers per plot for the whole field. PH was measured as the distance from the ground surface to the first spikelet of the spike.

\subsection{Genotyping}

\subsubsection{Whole genome shotgun sequencing of parental lines}

Genomic DNA of 21 Ae. tauschii accessions and six hexaploid parents used to create the introgression population was extracted from leaf tissues of two-week old seedlings grown in a greenhouse using DNeasy Plant Mini Kit (Qiagen) following the manufacturer's protocol. The quality and concentration of the DNA was assessed using PicoGreen dsDNA assay kit (Life Technologies).

Genomic libraries for Illumina sequencing were constructed from $\sim 2 \mu$ g of genomic DNA using the PCR-free Illumina protocol at the K-State Integrated Genomics Facility (IGF). The libraries were subjected to size selection using the Pippin Prep system (Sage Scientific) to enrich for 400-600 bp fragments. The pooled barcoded libraries were sequenced using the NovaSeq instrument $(2 \times 150$ bp run, S4 flow cell) at Kansas University Medical Center and NextSeq 500 (2 x 150 bp run) at IGF. The PCR-free whole genome shotgun sequencing libraries generated from 27 parental lines ranged between 200 and 700 bp with an average of 450 bp (Figure S1). Approximately 14 billion paired-end reads ( $150 \mathrm{bp}$ long) were generated from the libraries with an average of 0.54 billion reads per 
sample (data is available at NCBI SRA database BioPrject ID: PRJNA745927). The average number of reads per wheat line corresponded to approximately 10x genome coverage of parental lines. The raw reads with Phred quality score less than 15, minimum length less than $50 \mathrm{bp}$ and adaptor sequencies were filtered out using Trimmomatic v0.38-Java-1.8. The remaining filtered paired-end reads were mapped to the Chinese Spring (CS) RefSeq v1.0 (IWGSC, 2018) using BWA-mem software v0.7.17. A total of 7.1 billion reads were aligned uniquely to the CS RefSeq v1.0.

The sam files generated by BWA-mem were converted to bam files using SAMtools v1.10.

\subsubsection{Genotype imputation}

Sequence-based genotyping of the introgression population was performed previously by Nyine et al. (2020). SNPs with minor allele frequency (MAF) less than 0.01 were excluded from the raw vcf file using vcf-filter tools v0.1.13. The program conform-gt (https://faculty.washington.edu/ browning/conform-gt.html) was used to check the concordance of D genome SNP positions between the introgression population and the SNPs from parental lines genotyped by whole genome shotgun sequencing. Missing and ungenotyped SNPs in the D genome of the introgression population were imputed from the parental lines using Beagle v.5.0. A custom Perl script was used to filter out imputed SNPs with genotype probability below 0.7 and MAF less than 0.05 which resulted in 5.2 million SNPs. The filtered SNPs were used in the downstream analyses such as genome-wide association mapping and identification of the introgressed haplotype blocks.

\subsection{Introgressed haplotype detection}

Genetic divergence between the parental lines affects the probability of accurate detection of 
another one created by crossing hexaploid wheat with Ae. tauschii ssp. tauschii (Danby x TA2388, aka FAM97) to identify introgressed haplotype blocks. FAM93 had 21 introgression lines while FAM97 had 23 introgression lines. The R package HaploBlocker (Pook et al., 2019) was used to infer haplotype blocks from 5.2 million SNP sites. Recombinant inbred lines from each family were analyzed together with 21 Ae. tauschii and six hexaploid parents. The HaploBlocker parameters used in block calculation were node_min $=2$ (default is 5), overlap_remove $=$ TRUE, bp_map, equal_remove=TRUE. The parameter node_min was used to control the number of haplotypes per node during cluster-merging step of the block calculation function of HaploBlocker. The reduction in node_min was necessary to account for the low number of haplotype variants within these families. To maintain the SNP position in the haplotype block library, a vector of SNPs was provided via the parameter bp_map and prior to block calculation, SNPs in perfect linkage disequilibrium were removed by setting the parameter equal_remove = TRUE. Overlapping haplotypes were removed by setting parameter overlap_remove $=$ TRUE. Custom R and Perl scripts were used to calculate the haplotype block length using the information from haplotype block start and end coordinates. All monomorphic haplotypes between the two parental lines were excluded from the haplotype matrix before calculating the frequency of introgressed haplotypes per chromosome.

\subsection{Phenotype data analysis}

\subsubsection{Trait stability and heritability}

232

233

234

235

236

237

Yield stability is an important trait, which reflects the productivity of the crop under various growth conditions. No single statistic, however, is accurate enough to predict it due to high variability of genotype and genotype by environment interaction effects. In this study, we used 16 different statistics including parametric (such as Mean variance component $(\theta i)$, GE variance component $\left(\theta_{(i)}\right)$, Wricke's ecovalence $\left(W_{i}^{2}\right)$, Regression coefficient $\left(* b_{i}\right)$, Deviation from regression $\left(S_{d i}^{2}\right)$, Shukla's stability variance $\left(\sigma^{2}\right)$, Coefficient of variance $(C V i)$ and Kang's rank-sum (Kang or $K R)$ ) and non-parametric (such as Huhn's and Nassar and Huhn's $\left(S^{(1)}, S^{(2)}, S^{(3)}, S^{(4)}, S^{(5)}\right.$ and $S^{(6)}$ ) and Thennarasu's $\left(N P^{(1)}, N P^{(2)}, N P^{(3)}\right.$ and $\left.N P^{(4)}\right)$ ) methods to rank the introgression lines for yield stability based on their performance across years, locations and treatments. The description and properties of the statistics are documented at: https://manzik.com/stabilitysoft/. The analysis was implemented in R using a script from Pour-Aboughadareh et al. (2019) which is available at: https://github.com/pouraboughadareh/stabilitysoft. The most stable and/or high yielding lines were identified by sorting them according to their rankings.

Broad sense heritability $\left(H^{2}\right)$ and best linear unbiased predictions (BLUPs) for yield and the component traits were calculated from 2018 and 2019 data. A mixed linear model with restricted maximum likelihood implemented in $\mathrm{R}$ package lme 4 was used to generate the variance components (var) that were used to calculate heritability as follows.

Model $=\operatorname{lmer}($ Trait $\sim$ Trt $+(1 \mid$ Genotype $)+(1 \mid$ Genotype $:$ Trt $)$, data=trait_data $)$

$\mathrm{H}^{2}=\operatorname{var}($ Genotype $) /[\operatorname{var}($ Genotype $)+\operatorname{var}($ Genotype:Trt)/No. of Trt + var(Residual)/No. of Trt)]. mixed model as the random effects of genotypes.

Multiple comparison for the effect of treatment on yield and yield components traits in the introgression population relative to the controls was performed using least squares (LS) means with 'tukey' adjustment method and $\alpha=0.05$. To further assess the impact of introgressed haplotypes on the traits, lines were sorted in a descending order for each treatment and location/year. The 
percentage of the introgression lines that performed better than the best parental lines and checks both parents and checks were identified based on mean spatial adjusted yield. The percentage increase in yield was calculated as follows.

$$
\Delta \bar{y}_{i j}=100 \times \frac{\bar{y}_{i}-\bar{y}_{j}}{\bar{y}_{j}}
$$

Where $\Delta \bar{y}_{i j}$ is the percentage change in mean yield, $\bar{y}_{i}$ is the mean yield for the high yielding introgression lines and $\bar{y}_{j}$ is the mean yield for the controls (parents and checks).

\section{4}

265

266

267

268

\subsection{Trade-off between traits}

The relationship between yield, yield components and biomass traits were assessed by calculating the Pearson's correlation coefficients. We compared the trend of correlations from different treatments (irrigated/non-irrigated) and years to determine the extent of trade-off between traits within the introgression population and how the environment influenced them.

\subsection{Genome-wide association mapping}

Genome-wide association (GWAS) analysis was used to identify genomic regions with SNPs and haplotypes that have significant association with the traits. We tested the association of 5.2 million SNPs from the D genome with traits phenotyped in different treatments and years. A total of 15,967 haplotype block windows was identified from 5.2 million SNPs using the R package HaploBlocker v1.5.2 (Pook et al., 2019). Default parameters for HaploBlocker were used except node_min, which was reduced to 2 (default is 5) since most genomic intervals in our dataset had less than 5 haplotype variants. Overlapping haplotypes were removed using the parameter overlap_remove $=$ TRUE and the SNP coordinates were included in the haplotype library via the parameter bp_map. Haplotype blocks were split into windows by setting the parameter return_dataset $=$ TRUE in the block_windowdataset() function. The haplotype variants within a given chromosome interval were recorded as $0,1,2$ or 3 depending on the total number of haplotype variants present within the interval. In both cases, a mixed linear model implemented in R package GAPIT was used to detect the associations. To control for population structure in SNP-based analyses, 100,000 randomly selected markers were used to calculate the principal components. In haplotype-blockbased GWAS, all haplotype blocks were used to calculate the principal components. In both cases, only the first three principal components were used to control the population structure. Two threshold options were used to identify significant associations including a more stringent Bonferroni correction and a less stringent Benjamini and Hochberg (1995) false discovery rate (FDR) at 5\% significance level. To control for the effect of treatment and year, GWAS based on BLUPs was also performed and significant associations were reported only when there were SNP-trait or haplotypetrait association in at least two independent trials or in the BLUP-based analysis.

\subsection{SNP-trait and haplotype-trait effects}

Haplotype variation at loci with significant SNPs and their effects on traits in the introgression population were analyzed. The R package HaploBlocker v1.5.2 (Pook et al., 2019) was used to infer haplotypes at the genomic loci with significant SNP-trait associations. Heatmap.2 function provided in $\mathrm{R}$ package gplots was used to visualize the variation in haplotypes from hexaploid wheat and Ae. tauschii. However, at the Ppd-D1 locus, visual comparison of the SNPs from the parental lines was done and the SNPs were annotated using snpEff v4.3 software to resolve 
haplotype variants in the Ae. tauschii lines that could not be clearly distinguished by HaploBlocker. SNPs significant at FDR $\leq 0.05$ and their estimated allelic effects were selected from the association mapping results and used to verify if the haplotype effect corresponded to the observed phenotype in the introgression population. The mean and the standard deviation of the phenotype were calculated for each group of lines carrying a similar haplotype and the difference between the means was compared using Tukey's honestly significant difference and the student's $t$-test.

\section{$\begin{array}{lll}304 & 3 & \text { Results }\end{array}$}

\subsection{Trait variation in the introgression population}

Broad sense heritability $\left(\mathrm{H}^{2}\right)$ of GY was 0.7 in 2018 and 0.64 in 2019, while for the yield

The proportion of introgression lines outperforming the checks and parental lines for the measured traits varied between treatments and years with a minimum of $0.8 \%$ for BM in the nonirrigated trial in 2019 (CO19) and a maximum of 73\% for TGW in 2018 irrigated trial (COI18). The percentage increase in yield for the introgression lines that outperformed both checks and parents varied from $11 \%$ to 57\%, while the number of lines that produced more grains varied from 6 to 94 (Table 2). Under drought stress conditions in 2018 (CO18), the mean yield of top performing introgression lines was 1.6 and 1.4-folds higher than the checks and parents, respectively (Figure 1). These results suggest that some introgression lines carry alleles that confer drought tolerance thus ensuring high productivity under stressful conditions. The highest yield potential of both introgression lines and controls was observed in 2019.The mean of the top yielding introgression lines reached 134 bushels per acre (BPA) while that of the parental lines and checks reached 119 and $121 \mathrm{BPA}$, respectively (Table 2).

The yield stability analyses were performed to identify introgression lines that are both high yielding and stable under various environmental conditions. Ranking by mean yield showed that $6 \%$ of the lines carrying introgression produced more grains than most parental lines, except Larry (File S1). The yield of these lines ranged from 84.4 to $92.7 \mathrm{BPA}$. The average rank (AR) of 16 stability statistics revealed 12 lines with introgressions showing high yield stability. Five of these lines were both stable and high yielding according to Kang's rank-sum when compared to the controls. The yield of these five introgression lines (KS15SGDCB110-11, KS15SGDCB098-1, KS15SGDCB1036, KS15SGDCB098-14 and KS15SGDCB111-1) varied between 82 and 93 BPA. The yield of the most stable and high yielding introgression line (92.7 BPA) was $9.8 \%$ higher than the average yield of the controls (84.4 BPA). These results indicate that novel alleles from Ae. tauschii have the 
potential to increase the adaptive potential of hard red winter wheat to different environmental conditions. In addition, the stability statistics could help to prioritize introgression lines for deployment in different agroecological zones depending on their ranking in stability and yield. Lines that are moderately high yielding but show good yield stability could be deployed in marginal environments, whereas less stable but high yielding lines could be deployed in less stressful environments to achieve high productivity.

Harvest index (HI), a measure of source-sink capacity was also assessed for stability in

\subsection{Trade-off between yield and yield component traits}

Pearson's correlation coefficients between average yield and yield stability based on average rank (AR) of the 16 stability statistics was $-0.44(P<0.001)$, (File S1). However, the correlation between yield and Kang's rank-sum $(\mathrm{KR})$ was $-0.71(P<0.001)$, indicating that the most stable introgression lines were not necessarily the highest grain yielders, although there were some exceptions. Similarly, the correlation between average HI and AR was $-0.42(P<2.2 \mathrm{e}-16)$ while between $\mathrm{HI}$ and KR was $-0.73(P<2.2 \mathrm{e}-16)$, (File S2).

The trade-off between yield and yield components was influenced by the treatment, year and location as evidenced by the variation in the levels of correlations (File S3). Higher positive correlations were observed among grain morphometric traits such as TGW, GA, GW and GL, ranging from 0.13 (between GW and GL) to 0.96 (between TGW and GA), (Figure 2). HI and GSW positively correlated with GY, while the correlation between GY and GN was positive but nonsignificant in all trials, except for Colby irrigated trial in 2019 (COI19) (File S3). BM correlated negatively with HI, but showed a positive correlation with GSW (File S3). In some cases, increase in the SNS resulted in a reduction in the TGW, GA, GW or GL, consistent with the previously observed trade-off between these traits (Kuzay et al., 2019). In contrast, HD positively correlated with the SNS and $\mathrm{PH}$, which is in agreement with the previous findings (Shaw et al., 2013; Muqaddasi et al., 2019).

To further understand the contribution of different yield components to the final yield, we compared the phenotypes of the top yielding introgression lines to those of the controls across all treatments (File S4). In the CO18 trial under non-irrigated conditions, the introgression lines that outperformed the controls in yield had the highest TGW, GA and GL, whereas under irrigated conditions (COI18), all yield component traits showed the highest levels of expression in the top yielding introgression lines. The top yielding introgression lines in the CO19 trial had the highest HI, GW, SNS and BM, while TGW and GA were comparable to those of the parental lines. In the COI19 trial, the TGW, GA and GW traits contributed more towards the final yield compared to the GL, HI and BM traits. In the AS20 trial, high levels of heterogeneity were observed among the top yielding lines for the TGW, GA, GW and GL traits. However, these lines showed higher BM than the controls, resulting in a reduced $\mathrm{HI}$. impact on agronomic traits due to negative epistasis between the alleles of wild and cultivated wheat (Nyine et al., 2020). We investigated the relationship between the total size of introgressed genomic segments and phenotype. We found a positive linear relationship between GA, GL, SNS and the total 
386

387

388

389

390

391

392

393

394

395

396

397

398

399

400

401

402

403

404

405

406

407

408

409

410

411

412

413

414

415

416

417

418

419

420

421

422

423

424

425

426

427

428 size of the introgressed segments (Figure 3, File S5). For the TGW however, a positive linear relationship was only observed under drought stress conditions indicating that some wheat lines with large introgressions are efficient in utilizing the limited soil moisture and nutrients during grain filling. There was a negative relationship between GY, HI, GW, TGW under irrigated conditions and the size of introgression.

\subsection{Genotyping and imputation}

To identify Ae. tauschii haplotypes in the $\mathrm{D}$ genome of introgression population, we generated high-density SNP data. By whole-genome sequencing of six hexaploid parental lines and 21 Ae. tauschii accessions used for generating octoploid parents, we identified about 20 million high-quality SNP variants (MAF $\geq 0.05)$ and used them for genotype imputation in the introgression population genotyped by complexity-reduced sequencing. The total number of $\mathrm{D}$ genome SNPs retained after filtering out SNPs with genotype probability below 0.7 and MAF $<0.05$ was 5.2 million.

\subsection{Haplotypic variation between ssp. strangulata and ssp. tauschii families}

Using HaploBlocker v1.5.2, we identified 4,764 and 6,429 non-overlapping haplotype blocks in the Ae. tauschii ssp. strangulata (FAM93) and Ae. tauschii ssp. tauschii (FAM97) families, respectively. After filtering out the monomorphic haplotypes between the parental lines, $869(18 \%)$ and 3,020 (47\%) segregating haplotypes were retained in FAM93 and FAM97, respectively (Table 3, File S6). The low proportion of segregating haplotypes between hexaploid wheat and ssp. strangulata $\mathrm{D}$ genomes is in agreement with the finding that Ae. tauschii ssp. strangulata was the donor of the D genome of hexaploid wheat (Wang et al., 2013). These results also suggest that the high similarity between the genome of ssp. strangulata and the D genome of hexaploid wheat could result in underestimation of the proportion of introgressed haplotypes. The average genome-wide haplotype block length in FAM93 was higher (2 Mb) than that in the FAM97 (1 Mb), (File S6). There was a significant difference in the introgressed haplotype length between lines in FAM93 and FAM97 based on the t-test $(P=3.1 \mathrm{e}-16)$. The longest haplotype introgressed in all lines from FAM93 was $44 \mathrm{Mb}$ on chromosome arm 3DL while in FAM97 only four lines had a haplotype $>32$ $\mathrm{Mb}$ on chromosome arm 7DL. The number of segregating haplotypes in FAM93 varies from 32 (3D) to 336 (2D), while in FAM97 the number of segregating haplotypes varies from 173 (3D) to 617 (5D), (Table 3). In FAM93 and FAM97, the average frequency of each haplotype from Ae. tauschii parents in the introgression lines was 11 and 4, respectively (File S6).

\subsection{SNP- and haplotype-based genome-wide association mapping}

Genome-wide association study was performed in the Ae. tauschii introgression population to assess the effects of introgression into the $\mathrm{D}$ genome on the variance of traits related to biomass, yield and yield components and tenacious glume. The marker-trait association analyses were based on both individual SNPs and haplotype blocks identified by HaploBlocker from the 5.2 million imputed variants. We report only those associations that are replicated in at least two independent field trials and show significant association with both SNPs and haplotypes at FDR 0.05 (Table S1). Several genomic loci with significant associations distributed on the D genome chromosomes were detected for GL, GW and SNS. For other traits such as GY, TGW, GN, GA, HI, BM, GSW and SPSF, no consistent associations replicated in independent trials were detected.

We identified multiple significant SNP-trait and haplotype-trait associations from all trials on chromosome arms 2DS and 7DS for GL (Figure 4). The most significant SNPs were located in haplotype block windows 22,262,355 - 22,289,017 bp, 30,582,113 - 30,595,115 bp and 80,864,297- 
$81,398,316$ bp on chromosome arm 2DS, and 11,024,311 - 11,374,767 bp on chromosome arm 7DS (Table S1). Association analysis based on BLUPs confirmed the results from individual trials for GL on these two chromosomes. Other significant associations detected in at least two trials were identified on chromosome arms 3DS and 5DS (File S7).

We identified haplotypes with significant SNPs associated with GW on 1DL, 2DS, 6DL and 7DS from at least two independent trials that were confirmed by BLUP-based analysis (File S7). Haplotype block windows 65,964,778 - 66,124,103 bp and 66,265,325 - 66,266,089 bp showed the most significant association on 2DS.

At 95\% confidence level, the most significant SNP-trait associations were identified on

Previous studies have shown that SNS is linked to HD (Shaw et al., 2013; Muqaddasi et al., 2019). In the current study, we detected significant associations with SNS on chromosome arms 2DS and 6DL. We had one year data for HD and PH collected from Ashland in 2020, which provided us a good opportunity to validate this link in the Ae. tauschii introgression population. Genome-wide association mapping detected significant associations with HD on chromosome arms 2DS and 4DL while all D genome chromosomes showed significant association with PH but the strongest signals were observed on 1DS, 3DS and 6DL. The haplotype block window 16,548,753 - 16,639,561 bp on 2DS with the most significant SNPs for HD overlapped the locus showing significant association with SNS, which is in close proximity to another haplotype block overlapping with the most significant SNPs for SNS (16,497,666 - 16,548,006 bp). These results suggest that the expression of these two traits could be co-regulated.

For HD, the haplotype block windows on chromosome arm 4DL 442,735,095 - 442,751,954 bp and 459,271,685 - 459,290,731 bp had the most significant SNP-trait associations. The three traits (SNS, HD and PH) are known to be affected by the Rht8 and Ppd-Dl genes on 2DS, in addition to Rhtl on 4D, which controls plant height and flowering time (Borojevic and Borojevic 2005; Chen et al., 2018). Due to the lack of SNPs located near the Ppd-D1 gene locus at $\sim 34 \mathrm{Mb}(33,961,438-$ $33,951,651$ bp interval in CS RefSeq v1.0), we could not directly validate its association with these traits. However, significant associations for SNS were detected at $\sim 3 \mathrm{Mb}$ next to the Ppd-D1 locus in the CO19 and AS20 trials on haplotype blocks 2D:30,192,335 - 30,264,745 bp and 2D:28,829,778$28,937,705 \mathrm{bp}$, respectively. In the parental lines with high density SNPs ( 20 million), the Ppd-D1 locus had SNPs, which allowed us to precisely map the haplotypes from Ae. tauschii and hexaploid wheat lines. Results from HaploBlocker showed that all hexaploid parents carry an identical haplotype, which is distinct from that of Ae. tauschii accessions.

Using SNPs identified by whole-genome sequencing of parental lines, we characterized haplotypic diversity at the Ppd-D1 locus (Figure 5A). All hexaploid wheat lines carried the same Ppd-D1 haplotype (Hap1), while seven haplotypes of the Ppd-D1 gene (Hap2 -Hap8) were identified in Ae. tauschii. Whole genome sequencing of 21 Ae. tauschii revealed broader range of Ppd-D1 diversity compared to a previous study (Guo et al., 2009), which identified only three Ppd-D1 haplotypes. The Ae. tauschii ssp. strangulata accessions carried haplotypes that were identical to 
hexaploid wheat, except for Hap2 in TA1642, which had one SNP at position 33,952,131 bp (Figure 5A). The Ppd-Dl genic region in Ae. tauschii ssp. tauschii accessions has one synonymous (SN), three intronic (IN) and one missense (MS) SNPs. The missense variant at position 33,955,614 bp results in His16Asn change, which is predicted to have moderate functional impact, and only present in lines with haplotype Hap5 (Figure 5A). Next, we inferred the parental haplotypes of the Ppd-D1 locus in the introgression population by using SNPs within the $\sim 1-2 \mathrm{Mb}$ region surrounding the $P p d$ D1 locus. About $82 \%$ of the introgression lines carried haplotype Hap1.

Further, we evaluated linkage of $P p d-D 1$ haplotypes with SNP alleles showing significant and 2D_35558454, that flank the Ppd-D1 locus on both sides and have genotyping information in the introgression population. We compared them to SNP alleles that were significantly associated with SNS and HD in haplotype block window 2D: 16,548,753 - 16,639,561 bp (2D_16574050 and 2D_16574159), spanning $\sim 17 \mathrm{Mb}$ region (Figure 5B). We found that the GWAS alleles associated with increase in SNS and HD in the introgression population are also linked with two Ae. tauschii haplotypes (Hap_AeT** and Hap_AeT), whereas the GWAS alleles associated with decreasing effects were in LD with Hap_HW contributed by the hexaploid wheat parents. The Hap_AeT* group of haplotypes was contributed by the Ae. tauschii parents having Hap2 and Hap7 at the Ppd-D1 locus.

\subsection{The phenotypic effects of haplotype block variants}

\subsubsection{Average spikelet number per spike (SNS) and Heading date (HD)}

492

493

Significant haplotype-trait associations were identified on chromosome arms 2DS and 4DL that influence SNS and HD. Chromosome 2DS had multiple introgressed haplotypes that are significantly associated with the variation in SNS and HD with the most significant haplotypes located at $16,497,666-16,548,006$ bp and 16,548,753 - 16,639,561 bp for SNS and HD, respectively. The haplotype variants with the increasing effect at these loci were from Ae. tauschii parents while those with reducing effect were from the hexaploid wheat lines (Figure 5C, 5D). The verification of GWAS results for allelic effect at 2DS locus associated with SNS and HD supports the above observation (File S8). We observed a positive Pearson's correlation coefficient between SNS and HD; and lines having haplotypes from either parent showed significant differences in the phenotype based on a ttest ( $r=0.23, P=3.31 \mathrm{e}-07)$ at 95\% confidence level. Haplotypes on 4DL had smaller effect on SNS compared to HD. Among 35 and 66 introgression lines having SNS and HD trait values above the $90^{\text {th }}$ percentile of trait distribution, respectively, 13 lines had the increasing alleles from Ae. tauschii at both 2DS and 4DL loci associated with SNS and HD traits (Figure 5E).

Initially, we did not detect a significant GWAS signal directly associated with SNPs within the Ppd-Dl gene due to the lack of high-quality imputed SNPs in this region. Further analysis of parental haplotypes identified SNP variants linked with both the Ppd-DI haplotypes and haplotypes at $28 \mathrm{Mb}$ and $30 \mathrm{Mb}$ region showing the significant haplotype-trait association in two trials. These haplotypes were within $\sim 3 \mathrm{Mb}$ from the $P p d-D 1$ locus and likely overlap with $P p d-D 1$. We performed the analysis of variance to determine the effect of different haplotype variants identified in the parental accessions on the SNS in the introgression population using data from three experimental trials (COI19, CO19 and AS20) (Table 4). Results show that both hexaploid and Ae. tauschii haplotypes have a significant effect on SNS $(P<0.001)$ (Table 4, Figure 5B). Among the Ae. tauschii haplotypes, Hap7 had the highest impact on SNS $(P=0.003)$ followed by Hap2 $(P=0041)$ and Hap3 $(P=0.040)$. In contrast, Hap5 with the His16Asn missense mutation had a negative effect on SNS and was not significantly different from Hap1 present in hexaploid wheat lines $(P=0.072)$. These results are consistent with earlier studies that showed that the Ppd-D1 gene located at $\sim 34 \mathrm{Mb}$ 
518

519

520

(33,961,438 - 33,951,651 bp interval in CS RefSeq v.1.0), that plays a role in flowering time regulation in wheat also has a strong effect on variation in the SNS (Beales et al., 2007, Guo et al., 2009).

\subsubsection{Pleiotropic effects of haplotypes on yield component traits}

We also evaluated the effects of distinct haplotypes associated with HD on other traits. Haplotype Hap_AeT from chromosome 2D located at 16,548,753 - 16,639,561 bp is associated with significant increase in the days to heading and SNS without significant impact on BM, HI and GSW (Table 5).

We compared the effects of two haplotypes associated with GW on 2DS (2D: 65,964,778 66,124,103 bp and 2D: 66,265,325 - 66,266,089 bp), where Hap_HW from hexaploid parents and Hap_AeT_st from Ae.tauschii ssp. strangulata increase GW and Hap_AeT_ta from the Ae.tauschii ssp. tauschii parents reduces GW (Table 6). In Colby 2018 non-irrigated trial data, the haplotype at the 2D: $65,964,778-66,124,103$ bp locus that was associated with increase in GW and GL was also linked with increase in grain area, and decrease in grain number. While both Hap_AeT_st and Hap_AeT_ta haplotypes at 2D: 65,964,778 - 66,124,103 bp were associated with increase in grain length, only Hap_AeT_ta was linked with the significant increase in grain number (Table 6). The Hap_AeT_ta haplotype at the 2D: 66,265,325-66,266,089 bp haplotype block had similar effects on GN, although the observed difference was not significant.

Similarly, haplotype block 6D: 463,775,852 - 463,809,722 bp associated with PH and SNS has three variants (File S7). Haplotype variant Hap1_HW\&AeT is associated with increase in SNS, $\mathrm{PH}, \mathrm{BM}$ and GY. It showed no association with HD and grain traits, except grain number where an intermediate effect was observed. This haplotype variant is present in two Ae. tauschii lines (TA2389 and TA2398) and the hexaploid parents excluding KanMark and KS061862M-17. Haplotype variant Hap0_HW\&AeT, which is found in KanMark and KS061862M-17 and two ssp. strangulata accessions (TA1642 and TA2378) has an intermediate effect on GY, reducing it by 2 bushels compared to Hap1_HW\&AeT. The third haplotype variant (Hap_AeT) is present only in the Ae. tauschii lines.

The haplotype Hap_AeT contributed by Ae. tauschii at haplotype block 7D:14,185.651 $14,596,748 \mathrm{bp}$, was associated with significant increase in SNS compared to haplotypes present in winter wheat (Table S2). This increase was associated with significant decrease in GL and had no significant effect on GY. At 7D:14,722,457 - 14,817,138 bp, the Hap_AeT haplotype contributed by Ae. tauschii was also linked with significant increase in SNS compared to Hap1_HW\&AeT detected in both hexaploid wheat and Ae. tauschii parents. However, increase in SNS for this haplotype was connected with decrease in both GL and GY. At this haplotype block (7D:14,722,457 - 14,817,138 bp), the most significant increase in GY was observed for lines carrying haplotype Hap0_HW\&AeT, which was associated with moderate increase in both SNS and GL (Table S2).

\section{Discussion}

Here, we performed the sequence-based analysis of haplotypes in the wild-relative introgression population developed by crossing a diverse panel of Ae. tauschii accessions with winter wheat cultivars. Our results demonstrate that combining whole genome sequencing of wild and cultivated wheat founder lines with the complexity-reduced sequencing of a derived introgression population provides an effective framework for SNP imputation. Because most breeding populations are based on a limited number of founders, often including 10-30 lines, their whole genome 
sequencing is feasible in crops even with such large genomes as wheat, and provides a comprehensive description of allelic diversity present in a breeding population. The latter makes sequenced founders an ideal reference panel for imputing genotypes in a breeding population genotyped using low-coverage or complexity-reduced sequencing. This was recently demonstrated by imputing genotypes in the wheat MAGIC population genotyped by low-coverage sequencing (Scott et al., 2021). The composition of our introgression population including multiple bi-parental cross families (Nyine et al., 2020) also shifts the population allele frequency towards more common variants, which could be imputed with a higher accuracy than rare variants (Huang et al., 2015). In addition, the high levels of LD in the introgression population should increase the length of haplotype blocks and facilitate detection of matching haplotypes in the reference panel of founders using even sparse genotyping data generated by low-coverage or complexity-reducing sequencing. Consistent with these assumptions, an imputation algorithm implemented in Beagle (Browning and Browning 2013) allowed us to impute nearly 5.2 million SNPs in the introgression population with high genotype call probabilities above 0.7 using SNPs generated by complexity-reduced sequencing of this population and nearly 20 million variants identified in 27 founders. This high-density SNP marker data permitted detailed characterization of introgressed haplotypes (Pook et al., 2019) and assessing their effects on productivity traits.

Our results demonstrate that wild relative introgressions into the $\mathrm{D}$ genome of wheat, the least diverse amongst the three subgenomes, (Chao et al., 2010; Singh et al., 2019; Jordan et al., 2015) is associated with the increased levels of variation in yield and yield component traits. Analysis of data from several years and locations under irrigated and non-irrigated conditions revealed many superior introgression lines that produce more grains or show higher yield stability than the control cultivars. The yield increase in top-performing introgression lines was driven by a combination of yield component traits and in many cases, it was associated with increased grain size, grain weight, and biomass or improved harvest index. These results suggest that wild-relative introgression has the potential to positively affect source-sink balance, which was suggested to be one of the important factors contributing to yield potential (Reynolds et al., 2017). Many of these high yielding lines $(\sim 23 \%)$ were also among the top lines showing the highest levels of yield stability, indicating that introgression from Ae. tauschii likely improves the adaptive potential of hard red winter wheat in different environmental conditions. Consistent with this conclusion, the highest impact of introgression on yield was found in a non-irrigated trial, indicating that alleles from Ae. tauschii likely improve the adaption of hexaploid wheat to water-limiting conditions. The Ae. tasuchii accessions used to generate the introgression population represent both L1 and L2 lineages (Wang et al., 2013) and originate from a broad range of geographical locations with variable climatic conditions, likely capturing adaptive haplotypes from regions prone to drought stress.

Heading date is one of the key agronomic traits linked with wheat adaptation to different geographical locations and improvement in yield (Jung and Müller 2009). In our population, a positive correlation was observed between heading date and the spikelet number per spike with some lines showing up to two-week delay in heading date. Several haplotype blocks on chromosome arms 2DS and 4DL were significantly associated with variation in spikelet number and heading date. The haplotypes with increasing effects at both loci were derived from the Ae. tauschii indicating their potential for modulating both traits in bread wheat. Chromosome 2DS is known to harbor the Ppd-D1 and $R h t 8$ genes that control flowering time and plant height, respectively, and also could affect spikelet number (Shaw et al., 2013; Muqaddasi et al., 2019). The overlapping haplotype blocks associated with spikelet number and heading date were identified on 2DS, confirming that the two traits co-segregating in the population have a common genetic basis. We demonstrated that these 2DS haplotypes are associated with different allelic variants of the Ppd-Dl gene from Ae.tauschii. 
608

609

610

611

612

613

614

615

616

617

618

619

620

621

622

623

624

625

626

627

628

629

630

631

632

633

634

\section{5}

636

637

638

639

640

641

642

643

644

645

646

647

648

649

650

These results are consistent with the earlier studies that demonstrated that different alleles of the PpdDl gene have distinct effects on heading date and spikelet number per spike (Beales et al., 2007, Guo et al., 2009). These effects correlated with the relative expression levels of each Ppd-DI allele (Guo et al., 2009), suggesting that functional mutations within the $P p d-D 1$ coding region and the modifier mutations in the regulatory region of the gene likely account for the variation in these traits in the $A e$. tauschii-winter wheat introgression population. The developmental plasticity modulated by Ppd-DI is mediated by changes in the expression of flowering time genes (Gol et al. 2021). It was shown that the $\mathrm{Ppd}-\mathrm{Hl}$ from wild barley is capable of integrating environmental signals to control heading date and minimize the negative impact of transient drought stress on spikelet number (Gol et al. 2021). Consistent with this observation, in the current study, introgression lines that have a high proportion of Ae. tauschii segments produced more grain under drought stress in the Colby 2018 trial, raising the possibility that the Ae. tauschii alleles of Ppd-D1 also have the potential to protect wheat from the physiological effects of stress that lead to low yield.

Our study reveals that some haplotypes associated with productivity trait variation in the introgression population also exhibit significant pleiotropic effects. While the direction of effects on various traits was largely consistent with the previously reported trade-offs among component traits (Quintero et al., 2018; Griffiths et al., 2015; Reynolds et al., 2017), the combined effects of some introgressed haplotypes were associated with the positive trends in yield. For example, a haplotype contributed by Ae. tauschii ssp. tauschii at the chromosome 2D haplotype block at 65,964,778$66,124,103$ bp was associated with increase in grain length, size and number with moderate positive effect on grain yield. At the haplotype block on chromosome 7D located between 14,722,457 14,817,138 bp, the Hap0_HW\&AeT haplotype shared between hexaploid wheat and Ae. tauschii parents and associated with moderate increase in both spikelet number per spike and grain length was also associated with the most significant increase in grain yield. The analyses of pleotropic effects of introgressed haplotypes suggest that these haplotypes on chromosomes 2D and 7D could be utilized in breeding programs to improve yield component traits without negative effects on other productivity traits.

\section{Conclusions}

Imputation of markers from whole genome sequenced reference panels into skim-sequenced inference populations is increasingly becoming a common practice in plant breeding program due to its cost-effectiveness (Happ et al., 2019; Jessen et al., 2020). Our study demonstrates the utility of this strategy for detecting introgression in the wheat genome and contributes to developing genomic resources for deploying wild relative diversity in wheat breeding programs. We show that the haplotype-based analysis of trait variation in this population has the potential to improve our knowledge on the genetic effects of introgressed diversity on productivity traits and identify novel haplotypes for improving yield potential in wheat.

\section{Conflict of Interest}

The authors declare that the research was conducted in the absence of any commercial or financial relationships that could be construed as a potential conflict of interest.

\section{Author Contributions}

$\mathrm{AKF}$ and $\mathrm{MC}$ generated the Ae. tauschii introgression population. MN, EA, MC, RA, BB, WW, DD, $\mathrm{ZY}, \mathrm{YG}, \mathrm{FH}, \mathrm{KWJ}$ and AKF phenotyped the introgression population. MN, BB and AA generated the genotyping data. AA performed next-generation sequencing of parental lines and introgression 
651

652

653

654

655

656

657

658

659

660

661

662

663

664

665

666

667

668

669

670

671

672

673

674

675

676

677

678

679

680

681

682

683

684

685

686

687

688

689

690

691

692

693

population; $\mathrm{MN}$ and $\mathrm{FH}$ performed the bioinformatical analyses of data. MN performed the statistical analysis; EA conceived the idea and interpreted results; MN and EA wrote the manuscript. All coauthors contributed to the manuscript revision, read and approved the submitted version.

\section{$7 \quad$ Funding}

This research was supported by the Agriculture and Food Research Initiative Competitive Grants 2017-67007-25939 (WheatCAP) and 2020-68013-30905 from the USDA National Institute of Food and Agriculture, and by the International Wheat Yield Partnership (IWYP).

\section{Acknowledgments}

We would like to thank Mary Guttieri for providing access to the USDA ARS seed processing facility.

\section{$9 \quad$ References}

1. Bailey-Serres J, Parker JE, Ainsworth EA, Oldroyd GED, Schroeder JI. Genetic strategies for improving crop yields. Nature. 2019 Nov;575(7781):109.

2. Beales J, Turner A, Griffiths S, Snape JW, Laurie DA. A pseudo-response regulator is misexpressed in the photoperiod insensitive Ppd-Dla mutant of wheat (Triticum aestivum L.). Theoretical and Applied Genetics. 2007 Sep 1;115(5):721-33.

3. Benjamini Y, Hochberg Y. Controlling the false discovery rate: a practical and powerful approach to multiple testing. Journal of the Royal statistical society: series B (Methodological). 1995 Jan;57(1):289-300.

4. Borojevic K, Borojevic K. The Transfer and History of "Reduced Height Genes" (Rht) in Wheat from Japan to Europe. J Hered. 2005 Jun 1;96(4):455-9.

5. Browning BL, Browning SR. Improving the accuracy and efficiency of identity-by-descent detection in population data. Genetics. 2013 Jun 1;194(2):459-71.

6. Chao S, Dubcovsky J, Dvorak J, Luo M-C, Baenziger SP, Matnyazov R, et al., Population- and genome-specific patterns of linkage disequilibrium and SNP variation in spring and winter wheat (Triticum aestivum L.). BMC Genomics. 2010 Dec;11(1):1-17.

7. Chatzav M, Peleg Z, Ozturk L, Yazici A, Fahima T, Cakmak I, et al., Genetic diversity for grain nutrients in wild emmer wheat: potential for wheat improvement. Ann Bot. 2010 Jun 1;105(7):1211-20.

8. Chen L, Du Y, Lu Q, Chen H, Meng R, Cui C, Lu S, Yang Y, Chai Y, Li J, Liu L. The photoperiodinsensitive allele Ppd-D1a promotes earlier flowering in Rht12 dwarf plants of bread wheat. Frontiers in plant science. 2018 Oct 22;9:1312.

9. Dale Z, Jie H, Luyu H, Cancan Z, Yun Z, Yarui S, et al., An Advanced Backcross Population through Synthetic Octaploid Wheat as a "Bridge": Development and QTL Detection for Seed Dormancy. Front Plant Sci [Internet]. 2017 [cited 2021 Feb 8];8. Available from: https://www.frontiersin.org/articles/10.3389/fpls.2017.02123/full

10. Davies RW, Flint J, Myers S, Mott R. Rapid genotype imputation from sequence without reference panels. Nature genetics. 2016 Aug;48(8):965.

11. Del Moral LG, Rharrabti Y, Villegas D, Royo C. Evaluation of grain yield and its components in durum wheat under Mediterranean conditions: an ontogenic approach. Agronomy Journal. 2003 Mar 1;95(2):266-74.

12. Du Y, Cui B, Wang Z, Sun J, Niu W. Effects of manure fertilizer on crop yield and soil properties in China: A meta-analysis. Catena. 2020 Oct 1;193:104617. 
694

695

696

697

698

699

700

701

702

703

704

705

706

707

708

709

710

711

712

713

714

715

716

717

718

719

720

721

722

723

724

725

726

727

728

729

730

731

732

733

734

735

736

737

738

739

740
13. Dvorak J, Deal KR, Luo M-C, You FM, von Borstel K, Dehghani H. The Origin of Spelt and Free-Threshing Hexaploid Wheat. J Hered. 2012 May 1;103(3):426-41.

14. Dvořák J, Edge M, Ross K. On the evolution of the adaptation of Lophopyrum elongatum to growth in saline environments. PNAS. 1988 Jun 1;85(11):3805-9.

15. Gol L, Haraldsson EB, von Korff M. Ppd-H1 integrates drought stress signals to control spike development and flowering time in barley. J Exp Bot. 2021 Jan 20;72(1):122-36.

16. Golan G, Ayalon I, Perry A, Zimran G, Ade-Ajayi T, Mosquna A, et al., GNI - A1 mediates trade-off between grain number and grain weight in tetraploid wheat. Theor Appl Genet. 2019 Aug 1;132(8):2353-65.

17. Griffiths S, Wingen L, Pietragalla J, Garcia G, Hasan A, Miralles D, et al., Genetic Dissection of Grain Size and Grain Number Trade-Offs in CIMMYT Wheat Germplasm. PLOS ONE. 2015 Mar 16;10(3):e0118847.

18. Guo Z, Song Y, Zhou R, Ren Z, Jia J. Discovery, evaluation and distribution of haplotypes of the wheat Ppd-D1 gene. New Phytologist. 2009 Feb;185(3):841-51.

19. Hao M, Zhang L, Ning S, Huang L, Yuan Z, Wu B, et al., The Resurgence of Introgression Breeding, as Exemplified in Wheat Improvement. Frontiers in Plant Science [Internet]. 2020 [cited 2021 Feb 8];11. Available from: https://www.ncbi.nlm.nih.gov/pmc/articles/PMC7067975/

20. Happ MM, Wang H, Graef GL, Hyten DL. Generating High Density, Low Cost Genotype Data in Soybean [Glycine max (L.) Merr.]. G3: Genes, Genomes, Genetics. 2019 Jul 1;9(7):2153-60.

21. Hill WG, Robertson A. The effect of linkage on limits to artificial selection. Genetics Research. 1966 Dec;8(3):269-94.

22. Huang J, Howie B, McCarthy S, Memari Y, Walter K, Min JL, et al., Improved imputation of low-frequency and rare variants using the UK10K haplotype reference panel. Nat Commun. 2015 Sep 14;6(1):1-9.

23. Jensen SE, Charles JR, Muleta K, Bradbury PJ, Casstevens T, Deshpande SP, Gore MA, Gupta R, Ilut DC, Johnson L, Lozano R. A sorghum practical haplotype graph facilitates genome-wide imputation and cost-effective genomic prediction. The Plant Genome. 2020 Mar;13(1):e20009.

24. Jones BH, Blake NK, Heo H-Y, Kalous JR, Martin JM, Torrion JA, et al., Improving hexaploid spring wheat by introgression of alleles for yield component traits from durum wheat. Crop Science. 2020 Mar 1;60(2):759-71.

25. Jordan KW, Wang S, Lun Y, Gardiner L-J, MacLachlan R, Hucl P, et al., A haplotype map of allohexaploid wheat reveals distinct patterns of selection on homoeologous genomes. Genome Biol. 2015 Dec;16(1):1-18.

26. Jung C, Müller AE. Flowering time control and applications in plant breeding. Trends in plant science. 2009 Oct 1;14(10):563-73.

27. Kuzay S, Xu Y, Zhang J, Katz A, Pearce S, Su Z, et al., Identification of a candidate gene for a QTL for spikelet number per spike on wheat chromosome arm 7AL by high-resolution genetic mapping. TAG Theoretical and Applied Genetics Theoretische Und Angewandte Genetik. 2019;132(9):2689.

28. Li Y, Willer CJ, Ding J, Scheet P, Abecasis GR. MaCH: using sequence and genotype data to estimate haplotypes and unobserved genotypes. Genetic epidemiology. 2010 Dec;34(8):816-34.

29. Lopes MS, El-Basyoni I, Baenziger PS, Singh S, Royo C, Ozbek K, et al., Exploiting genetic diversity from landraces in wheat breeding for adaptation to climate change. J Exp Bot. 2015 Jun $1 ; 66(12): 3477-86$.

30. Lorenz AJ, Hamblin MT, Jannink J-L. Performance of Single Nucleotide Polymorphisms versus Haplotypes for Genome-Wide Association Analysis in Barley. PLOS ONE. 2010 Nov 22;5(11):e14079. 
31. McKenna A, Hanna M, Banks E, Sivachenko A, Cibulskis K, Kernytsky A, et al., The Genome Analysis Toolkit: A MapReduce framework for analyzing next-generation DNA sequencing data. Genome Res. 2010 Jan 9;20(9):1297-303.

32. Mulitze DK. AGROBASE/4: A microcomputer database management and analysis system for plant breeding and agronomy. Agronomy Journal. 1990 Sep;82(5):1016-21.

33. Muqaddasi QH, Brassac J, Koppolu R, Plieske J, Ganal MW, Röder MS. TaAPO-A1, an ortholog of rice ABERRANT PANICLE ORGANIZATION 1, is associated with total spikelet number per spike in elite European hexaploid winter wheat (Triticum aestivum L.) varieties. Sci Rep. 2019 Sep 25;9(1):1-12.

34. Nyine M, Adhikari E, Clinesmith M, Jordan KW, Fritz AK, Akhunov E. Genomic patterns of introgression in interspecific populations created by crossing wheat with its wild relative. G3: Genes, Genomes, Genetics. 2020 Oct 1;10(10):3651-61.

35. Nyine M, Wang S, Kiani K, Jordan K, Liu S, Byrne P, Haley S, Baenziger S, Chao S, Bowden R, Akhunov E. Genotype imputation in winter wheat using first-generation haplotype map SNPs improves genome-wide association mapping and genomic prediction of traits. G3: Genes, Genomes, Genetics. 2019 Jan 1;9(1):125-33.

36. Pasquariello M, Berry S, Burt C, Uauy C, Nicholson P. Yield reduction historically associated with the Aegilops ventricosa 7D V introgression is genetically and physically distinct from the eyespot resistance gene Pch1. Theor Appl Genet. 2020 Mar 1;133(3):707-17.

37. Pennacchi JP, Carmo-Silva E, Andralojc PJ, Lawson T, Allen AM, Raines CA, Parry MA. Stability of wheat grain yields over three field seasons in the UK. Food and energy security. 2019 May;8(2):e00147.

38. Periyannan S, Moore J, Ayliffe M, Bansal U, Wang X, Huang L, et al., The Gene Sr33, an Ortholog of Barley Mla Genes, Encodes Resistance to Wheat Stem Rust Race Ug99. Science. 2013 Aug 16;341(6147):786-8.

39. Placido DF, Campbell MT, Folsom JJ, Cui X, Kruger GR, Baenziger PS, et al., Introgression of Novel Traits from a Wild Wheat Relative Improves Drought Adaptation in Wheat. Plant Physiology. 2013 Apr;161(4):1806.

40. Pook T, Schlather M, Campos G de los, Mayer M, Schoen CC, Simianer H. HaploBlocker: Creation of Subgroup-Specific Haplotype Blocks and Libraries. Genetics. 2019 Aug 1;212(4):1045-61.

41. Pour-Aboughadareh A, Yousefian M, Moradkhani H, Poczai P, Siddique KHM. STABILITYSOFT: A new online program to calculate parametric and non-parametric stability statistics for crop traits. Appl Plant Sci. 2019 Jan;7(1):e01211.

42. Quintero A, Molero G, Reynolds MP, Calderini DF. Trade-off between grain weight and grain number in wheat depends on GxE interaction: A case study of an elite CIMMYT panel (CIMCOG). Eur J Agron [Internet]. 2018;92:17-29. Available from: http://www.sciencedirect.com/science/article/pii/S1161030117301375

43. Reynolds M, Chapman S, Crespo-Herrera L, Molero G, Mondal S, Pequeno DNL, et al., Breeder friendly phenotyping. Plant Science 2020 Jun 18;295:110396.

44. Reynolds MP, Calderini DF, Condon AG, Rajaram S. Physiological basis of yield gains in wheat associated with the LR19 translocation from Agropyron elongatum. Euphytica. 2001 May 1;119(1):139-44.

45. Reynolds MP, Pask AJD, Hoppitt WJE, Sonder K, Sukumaran S, Molero G, et al., Strategic crossing of biomass and harvest index-source and sink-achieves genetic gains in wheat. Euphytica. 2017 Nov 1;213(11):1-23.

46. Saintenac C, Zhang W, Salcedo A, Rouse MN, Trick HN, Akhunov E, et al., Identification of Wheat Gene Sr35 that Confers Resistance to Ug99 Stem Rust Race Group. Science (New York, NY). 2013 Aug 16;341(6147):783. 
47. Sears RG. Strategies for improving wheat grain yield. Wheat: Prospects for Global Improvement. 1997;17-21

48. Scott MF, Fradgley N, Bentley AR, Brabbs T, Corke F, Gardner KA, et al., Limited haplotype diversity underlies polygenic trait architecture across 70 years of wheat breeding. Genome Biol. 2021 May 6;22(1):137.

49. Singh N, Wu S, Tiwari V, Sehgal S, Raupp J, Wilson D, et al., Genomic Analysis Confirms Population Structure and Identifies Inter-Lineage Hybrids in Aegilops tauschii. Front Plant Sci [Internet]. 2019 [cited 2021 Feb 10];10. Available from: https://www.frontiersin.org/articles/10.3389/fpls.2019.00009/full

50. Shaw LM, Turner AS, Herry L, Griffiths S, Laurie DA. Mutant alleles of Photoperiod-1 in wheat (Triticum aestivum L.) that confer a late flowering phenotype in long days. PLoS One. 2013 Nov 14;8(11):e79459.

51. Swarts K, Li H, Romero Navarro JA, An D, Romay MC, Hearne S, et al., Novel Methods to Optimize Genotypic Imputation for Low-Coverage, Next-Generation Sequence Data in Crop Plants. Plant Genome [Internet]. 2014;7:1-12. Available from: https://www.crops.org/publications/tpg/abstracts/7/3/plantgenome2014.05.0023

52. Wang J, Luo MC, Chen Z, You FM, Wei Y, Zheng Y, Dvorak J. Aegilops tauschii single nucleotide polymorphisms shed light on the origins of wheat D-genome genetic diversity and pinpoint the geographic origin of hexaploid wheat. New phytologist. 2013 May;198(3):925-37

53. Wang RR-C, Zhang X-Y. Characterization of the translocated chromosome using fluorescence in situ hybridization and random amplified polymorphic DNA on two Triticum aestivumThinopyrum intermedium translocation lines resistant to wheat streak mosaic or barley yellow dwarf virus. Chromosome Res. 1996 Dec 1;4(8):583-7.

54. Wang S, Wong D, Forrest K, Allen A, Chao S, Huang BE, et al., Characterization of polyploid wheat genomic diversity using a high-density 90000 single nucleotide polymorphism array. Plant Biotechnology Journal. 2014 Aug;12(6):787.

55. Zhang H, Mittal N, Leamy LJ, Barazani O, Song B-H. Back into the wild-Apply untapped genetic diversity of wild relatives for crop improvement. Evolutionary Applications. 2017 Jan 1;10(1):5-24.

56. Zhang J, Gizaw SA, Bossolini E, Hegarty J, Howell T, Carter AH, et al., Identification and validation of QTL for grain yield and plant water status under contrasting water treatments in fallsown spring wheats. TAG Theoretical and Applied Genetics 2018;131(8):1741.

57. Zhang K, Calabrese P, Nordborg M, Sun F. Haplotype block structure and its applications to association studies: power and study designs. The American Journal of Human Genetics. 2002 Dec 1;71(6):1386-94. 
838 Table 1. Comparison of grain yield between introgression lines (progeny) and controls

839 (checks/hexaploid parents) per treatment within a year using least squares (LS) means.

\begin{tabular}{lllcccccc}
\hline Year & Treatment & Group & LSmean & SE & df & lower.CL & upper.CL & group \\
\hline 2018 & Irrigated & Progeny & 68.6 & 0.77 & 410 & 66.8 & 70.5 & $\mathrm{a}$ \\
2018 & Irrigated & Check & 72.1 & 7.64 & 410 & 53.8 & 90.4 & $\mathrm{a}$ \\
2018 & Irrigated & Parent & 75.7 & 3.6 & 410 & 67.1 & 84.3 & $\mathrm{a}$ \\
2018 & Non-irrigated & Progeny & 50.1 & 6.43 & 395 & 34.6 & 65.5 & $\mathrm{a}$ \\
2018 & Non-irrigated & Check & 51.6 & 0.66 & 395 & 50 & 53.2 & $\mathrm{a}$ \\
2018 & Non-irrigated & Parent & 57.1 & 2.95 & 395 & 50 & 64.2 & $\mathrm{a}$ \\
2019 & Irrigated & Progeny & 103 & 0.82 & 379 & 100.6 & 105 & $\mathrm{a}$ \\
2019 & Irrigated & Parent & 119 & 3.8 & 379 & 109.5 & 128 & $\mathrm{~b}$ \\
2019 & Irrigated & Check & 121 & 9.05 & 379 & 99.3 & 143 & $\mathrm{ab}$ \\
2019 & Non-irrigated & Progeny & 101 & 0.86 & 382 & 99.2 & 103 & $\mathrm{a}$ \\
2019 & Non-irrigated & Parent & 116 & 3.85 & 382 & 106.3 & 125 & $\mathrm{~b}$ \\
2019 & Non-irrigated & Check & 116 & 9.44 & 382 & 93.5 & 139 & $\mathrm{ab}$ \\
2020 & Non-irrigated & Progeny & 47.1 & 0.43 & 385 & 46 & 48.1 & $\mathrm{a}$ \\
2020 & Non-irrigated & Parent & 55.3 & 2.01 & 385 & 50.5 & 60.1 & $\mathrm{~b}$ \\
2020 & Non-irrigated & Check & 59.3 & 4.79 & 385 & 47.8 & 70.8 & $\mathrm{~b}$ \\
\hline
\end{tabular}

Note: The 2018 and 2019 trials were conducted at Colby while the 2020 trial was conducted at Ashland, Kansas State. 
860 Table 2. Percentage mean yield difference between top performing introgression lines and 861 controls (parents and checks) per treatment in each year and location.

\begin{tabular}{lcccc}
\hline \multicolumn{1}{c}{ Group ID } & No. of IL & Mean yield IL & Mean yield controls & \% yield diff. \\
\hline AS20_ILP & 6 & 68.6 & 55.2 & 24.3 \\
AS20_ILC & 6 & 68.6 & 59.3 & 15.8 \\
CO18_ILP & 21 & 78.7 & 57.1 & 37.8 \\
CO18_ILC & 21 & 78.7 & 50.1 & 57.1 \\
COI18_ILP & 94 & 86.9 & 75.7 & 14.8 \\
COI18_ILC & 94 & 86.9 & 72.1 & 20.6 \\
CO19_ILP & 11 & 130.5 & 115.5 & 13.0 \\
CO19_ILC & 11 & 130.5 & 116.1 & 12.4 \\
COI19_ILP & 6 & 134.2 & 119.2 & 12.6 \\
COI19_ILC & 6 & 134.2 & 121.0 & 10.9 \\
\hline
\end{tabular}

862 AS: Ashland non-irrigated trial, CO: Colby non-irrigated trial, COI: Colby irrigated trial, ILP: introgression lines by

863 parents' grain yield comparison and ILC: introgression lines by checks grain yield comparison. 

and Ae. tauschii ssp. tauschii (FAM97) families.

\begin{tabular}{llllllll}
\hline Family & Pedigree & Subspecies & Chrom & NH & MHL (bp) & MHF & Range (bp) \\
\hline FAM93 & KanMark x TA1642 & strangulata & chr1D & 105 & $1,896,815$ & 4.6 & $1,788-8,639,976$ \\
FAM93 & KanMark x TA1642 & strangulata & chr2D & 336 & $1,326,620$ & 20.0 & $813-30,656,640$ \\
FAM93 & KanMark x TA1642 & strangulata & chr3D & 32 & $6,653,571$ & 4.9 & $2,525-44,264,371$ \\
FAM93 & KanMark x TA1642 & strangulata & chr4D & 143 & $1,865,609$ & 4.3 & $2,942-12,070,089$ \\
FAM93 & KanMark x TA1642 & strangulata & chr5D & 138 & $2,302,767$ & 7.1 & $556-15,103,840$ \\
FAM93 & KanMark x TA1642 & strangulata & chr6D & 65 & $2,519,945$ & 3.0 & $3,455-13,483,300$ \\
FAM93 & KanMark x TA1642 & strangulata & chr7D & 50 & $2,667,416$ & 2.04 & $1,627-11,349,447$ \\
FAM97 & Danby x TA2388 & tauschii & chr1D & 495 & 829,609 & 3.1 & $762-9,169,049$ \\
FAM97 & Danby x TA2388 & tauschii & chr2D & 352 & $1,305,439$ & 1.8 & $813-15,991,790$ \\
FAM97 & Danby x TA2388 & tauschii & chr3D & 173 & $2,968,356$ & 3.0 & $884-27,225,468$ \\
FAM97 & Danby x TA2388 & tauschii & chr4D & 540 & 750,352 & 0.8 & $1,982-20,010,593$ \\
FAM97 & Danby x TA2388 & tauschii & chr5D & 617 & 770,483 & 10.1 & $1,243-15,396,133$ \\
FAM97 & Danby x TA2388 & tauschii & chr6D & 373 & 830,168 & 3.8 & $764-23,208,199$ \\
FAM97 & Danby x TA2388 & tauschii & chr7D & 465 & $1,000,899$ & 3.7 & $1,397-32,547,936$ \\
\hline
\end{tabular}


902 Table 4. Analysis of variance for the effect of Ppd-Dl haplotype variants from hexaploid wheat and

903 Ae. tauschii on the spikelet number per spike in three experimental trials of the introgression

904 population.

\begin{tabular}{lrrrc}
\hline Haplotype & Estimate & Std. Error & t value & $\operatorname{Pr}(>|\mathbf{t}|)$ \\
\hline Hexaploid Wheat (HW) & 13.38 & 0.13 & -3.41 & $0.000677^{* * *}$ \\
Ae. tauschii (AeT) & 13.83 & 0.12 & 115.84 & $<2 \mathrm{e}-16^{* * *}$ \\
HW_Hap1 & 13.38 & 0.84 & 1.79 & 0.07317 \\
AeT_Hap2 & 13.79 & 0.94 & 2.05 & $0.04092^{*}$ \\
AeT_Hap3 & 13.74 & 0.91 & 2.06 & $0.03958^{*}$ \\
AeT_Hap4 & 13.50 & 1.19 & 1.37 & 0.17002 \\
AeT_Hap5 & 13.53 & 0.92 & 1.80 & 0.07190 \\
AeT_Hap6 & 13.69 & 0.93 & 1.96 & 0.05069 \\
AeT_Hap7 & $\mathbf{1 4 . 6 2}$ & $\mathbf{0 . 9 2}$ & $\mathbf{2 . 9 9}$ & $\mathbf{0 . 0 0 2 8 8 * *}$ \\
AeT_Hap8 & 13.77 & 1.19 & 1.60 & 0.11051 \\
\hline
\end{tabular}

905

Significance codes $* * *=0.001, * *=0.01, *=0.05$

906

907

908

909

910

911

912

913

914

915

916

917

918

919

920

921

922

923

924

925

926

927

928

929

930

931

932

933 
934 Table 5. Chromosome 2D haplotypes variants associated with the spikelet number and heading 935 date and how they influence other traits in the introgression population.

\begin{tabular}{llccc}
\hline Trait & Haplotype & Loc1 Freq. & chr2D:16548753-16639561 & SD \\
\hline HD & Hap_HW & 53 & $199^{\mathrm{b}}$ & $2.28 *$ \\
HD & Hap_AeT & $\mathbf{1 5 6}$ & $\mathbf{2 0 2}^{\mathrm{a}}$ & $\mathbf{2 . 8 6}$ \\
HD & Hap_HW/AeT & 142 & $200^{\mathrm{c}}$ & $2.29+$ \\
PH & Hap_HW & 53 & $68.27^{\mathrm{ab}}$ & 6.79 \\
PH & Hap_AeT & 156 & $70.82^{\mathrm{a}}$ & 6.97 \\
PH & Hap_HW/AeT & 142 & $65.9^{\mathrm{b}}$ & 8.68 \\
BM & Hap_HW & 53 & $155.18^{\mathrm{a}}$ & 28.21 \\
BM & Hap_AeT & 156 & $156.26^{\mathrm{a}}$ & 31.01 \\
BM & Hap_HW/AeT & 142 & $157.52^{\mathrm{a}}$ & 35.82 \\
SNS & Hap_HW & 53 & $14.72^{\mathrm{b}}$ & 1.2 \\
SNS & Hap_AeT & $\mathbf{1 5 6}$ & $\mathbf{1 5 . 2 7 ^ { \mathrm { a } }}$ & $\mathbf{1 . 1 2}$ \\
SNS & Hap_HW/AeT & 142 & $14.05^{\mathrm{c}}$ & 1.03 \\
GSW & Hap_HW & 53 & $66.84^{\mathrm{a}}$ & 14.71 \\
GSW & Hap_AeT & 156 & $65.82^{\mathrm{a}}$ & 13.67 \\
GSW & Hap_HW/AeT & 142 & $66.47^{\mathrm{a}}$ & 13.57 \\
HI & Hap_HW & 53 & $42.85^{\mathrm{a}}$ & 4.35 \\
HI & Hap_AeT & 156 & $42.23^{\mathrm{a}}$ & 4.24 \\
HI & Hap_HW/AeT & 142 & $42.89^{\mathrm{a}}$ & 6.28 \\
\hline
\end{tabular}
date, $\mathbf{P H}$ is average plant height, BM is aboveground total dry biomass, SNS is spikelet number per spike, GSW is Grain

939 sample weight from biomass sample and $\mathbf{H I}$ is harvest index. Means with the same superscript letters are not statistically 
bioRxiv preprint doi: https://doi.org/10.1101/2021.05.29.446303; this version posted July 15, 2021. The copyright holder for this preprint (which

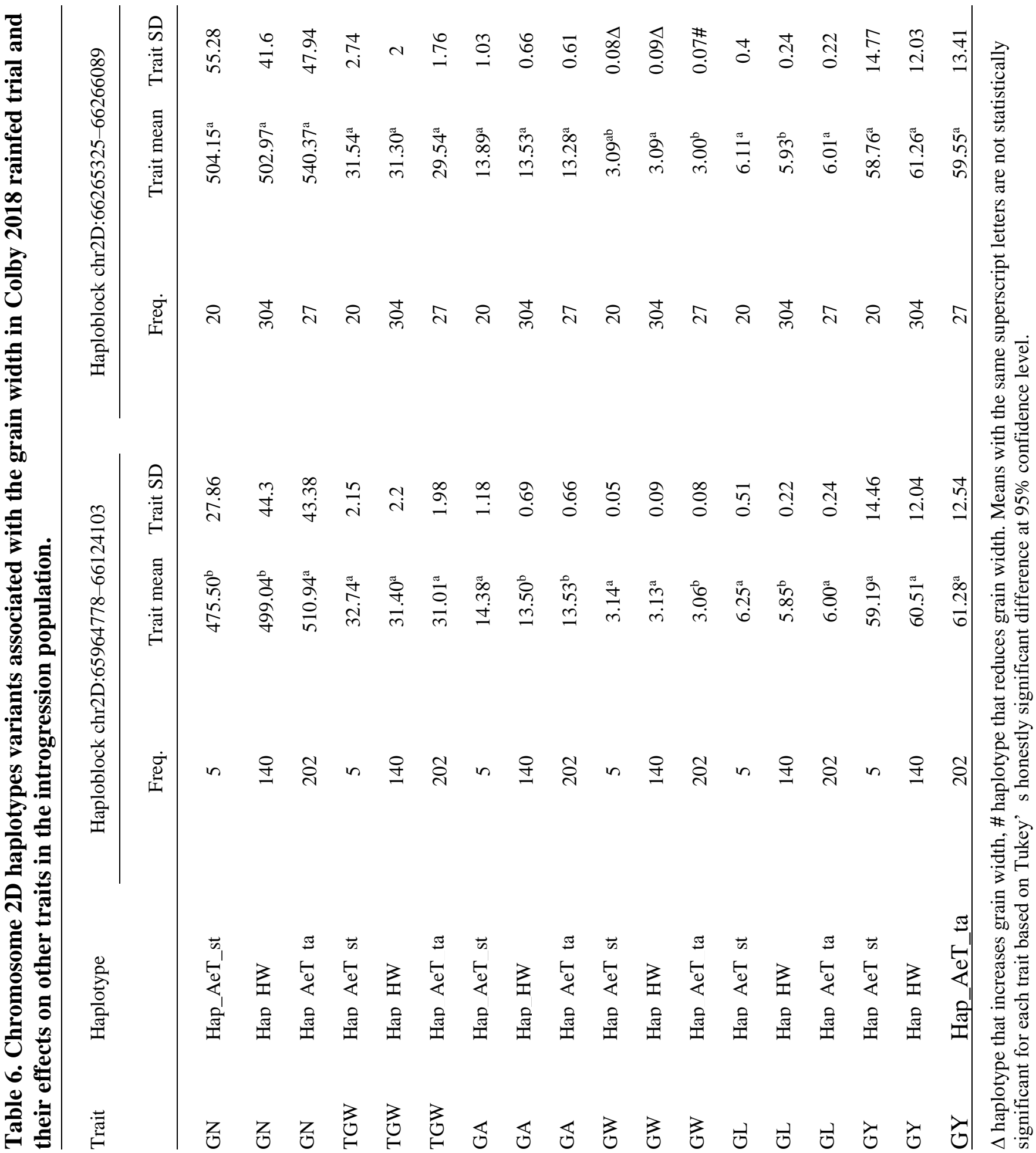




\section{Figure caption}

960

961

962

963

964

965

966

967

968

969

970

971

972

973

974

975

976

977

978

979

980

981

982

983

984

985

986

987

988

989

990

991

992

993

994

995

996

997

998

999

Figure 1. Boxplots comparing the mean grain yield between the top performing introgression lines (IL) and the controls (parents and checks) across treatments, years and locations. Where AS20 refers to Ashland rainfed trial in 2020, CO18 is Colby rainfed trial in 2018, COI18 is Colby irrigated trial in 2018, CO19 is Colby rainfed trial in 2019 and COI19 is Colby irrigated trial in 2019.

Figure 2. Pearson's correlation coefficients between yield and yield component traits at Colby in 2018 rainfed trial (A) and Ashland in 2020 rainfed trial (B). Where HD is heading date, PH is plant height, BM is aboveground dry biomass, SPSF is spikes per square foot, SPB is spikes per bag, SNS is spikelet number per spike, GSW is grain sample weight, $\mathbf{H I}$ is harvest index, $\mathbf{G N}$ is grain number, TGW is thousand grain weight, GA is grain area, GW is grain width, GL is grain length, GY is grain yield.

Figure 3. Relationship between spikelet number per spike (A \& B), grain length $(\mathbf{C} \& \mathbf{D})$, grain yield $(\mathbf{E} \& \mathbf{F})$ and the proportion of introgression under non-irrigated conditions at Colby in 2019 (CO19) and Ashland in 2020 (AS20). Here, $\mathbf{r}$ is the correlation coefficient and $\mathbf{P}$ is the significance of the correlation between introgression size and observed trait phenotype.

Figure 4. Manhattan plots showing the D genome loci with SNPs and haplotypes that are significantly associated with spikelet number per spike at Ashland in 2020 (AS20) under rainfed conditions (A \& B) and grain length at Colby in 2018 (COI18) under irrigated conditions (C \& D) in the Ae. tauschii introgression population. The horizontal solid black line shows a threshold of 0.05 significance level for Bonferroni correction, the black arrowheads indicate the SNPs and haplotypes above the threshold.

Figure 5. Effect of haplotypes introgressed from Ae. tauschii into the chromosome arms 2DS and 4DL of hexaploid wheat on the spikelet number per spike (SNS) and heading date (HD) of the introgression lines and the possible link to Ppd-D1 gene located on 2DS. (A) SNP table showing the haplotype variants at the $P p d-D 1$ gene locus in winter wheat accessions (top six) and the $21 A e$. tauschii lines. The black rectangle shows SNPs within the coding region of the gene where $\mathbf{S N}$ is a synonymous SNP, IN is an intronic SNP and MS is a missense SNP (His16Asn) as reported by snpEff v4.3 software. (B) Unique haplotypes in the introgression population tagging the Ppd-D1 locus and GWAS signal for SNS and HD on chromosome arm 2D, and the associated phenotype. Hap_HW is present in introgression lines that have Hap1 from hexaploid wheat at the Ppd-D1 locus, Hap_AeT includes lines that have Hap3-6 and 8 while Hap_AeT* includes lines that have Hap2 and Hap7 at the Ppd-D1 locus in Ae. tauschii parents. The TT and CA alleles at the GWAS signal have reducing and increasing effects, respectively, on SNS and HD. (C) Boxplot showing the impact of introgression from Ae. tauschii in chromosome arms 2DS and 4DL on SNS. (D) Boxplot showing the impact of introgression from Ae. tauschii in chromosome arms 2DS and 4DL on HD. (E) A Venn diagram showing the number of introgression lines in the 90th percentile for SNS and HD. Lines in the intersection have the increasing alleles on both 2DS and 4DL loci associated with SNS and HD. $* * *$ indicates significant difference between groups with $\mathrm{P}<0.001$ while NS indicates a nonsignificant difference based on t-test statistics. 
bioRxiv preprint doi: https://doi.org/10.1101/2021.05.29.446303; this version posted July 15, 2021. The copyright holder for this preprint (which

was not certified by peer review) is the author/funder, who has granted bioRxiv a license to display the preprint in perpetuity. It is made available under aCC-BYGehleticeatifectseofe.Ae. tauschii-wheat introgression

1001

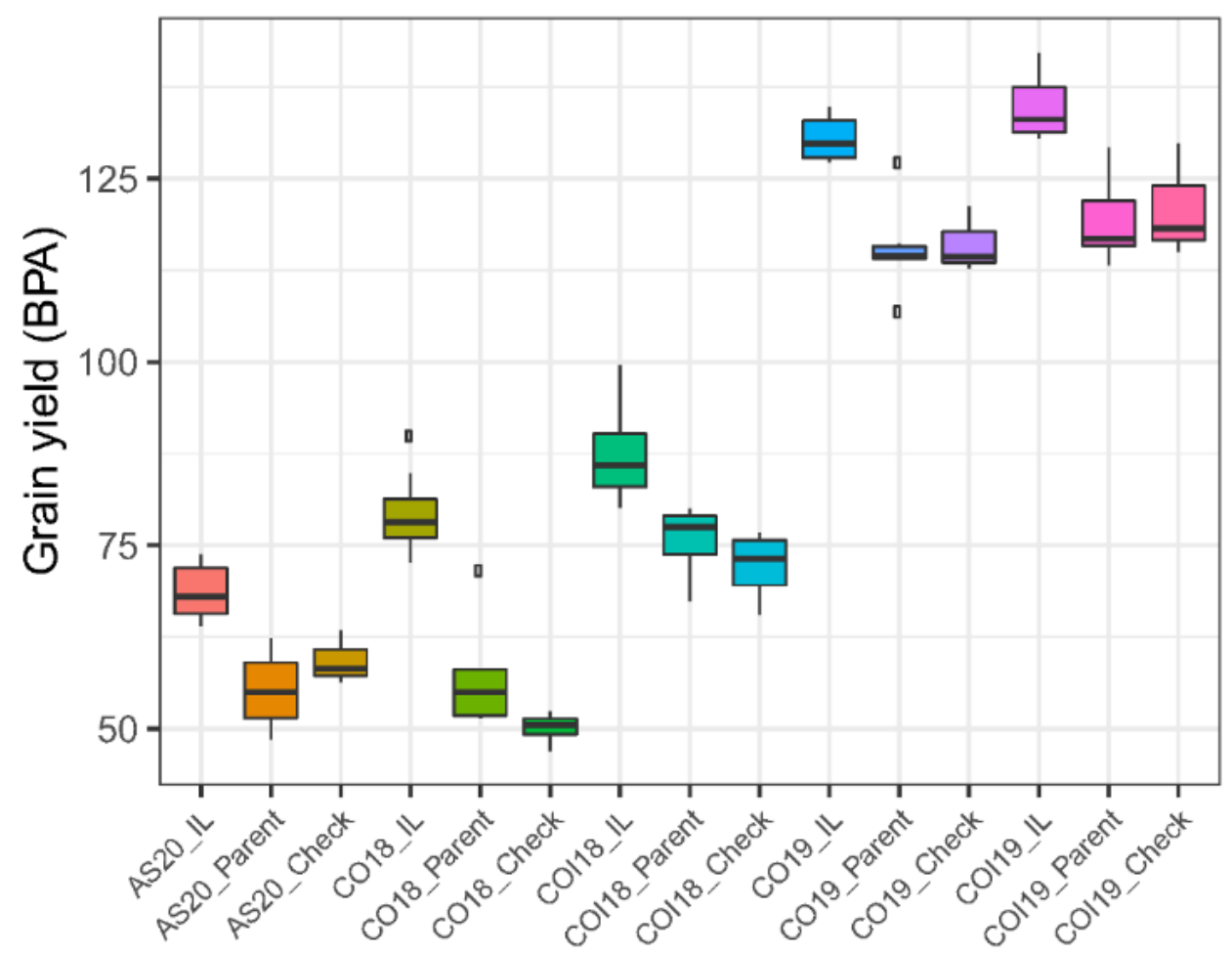

Fig. 1

1003

1004
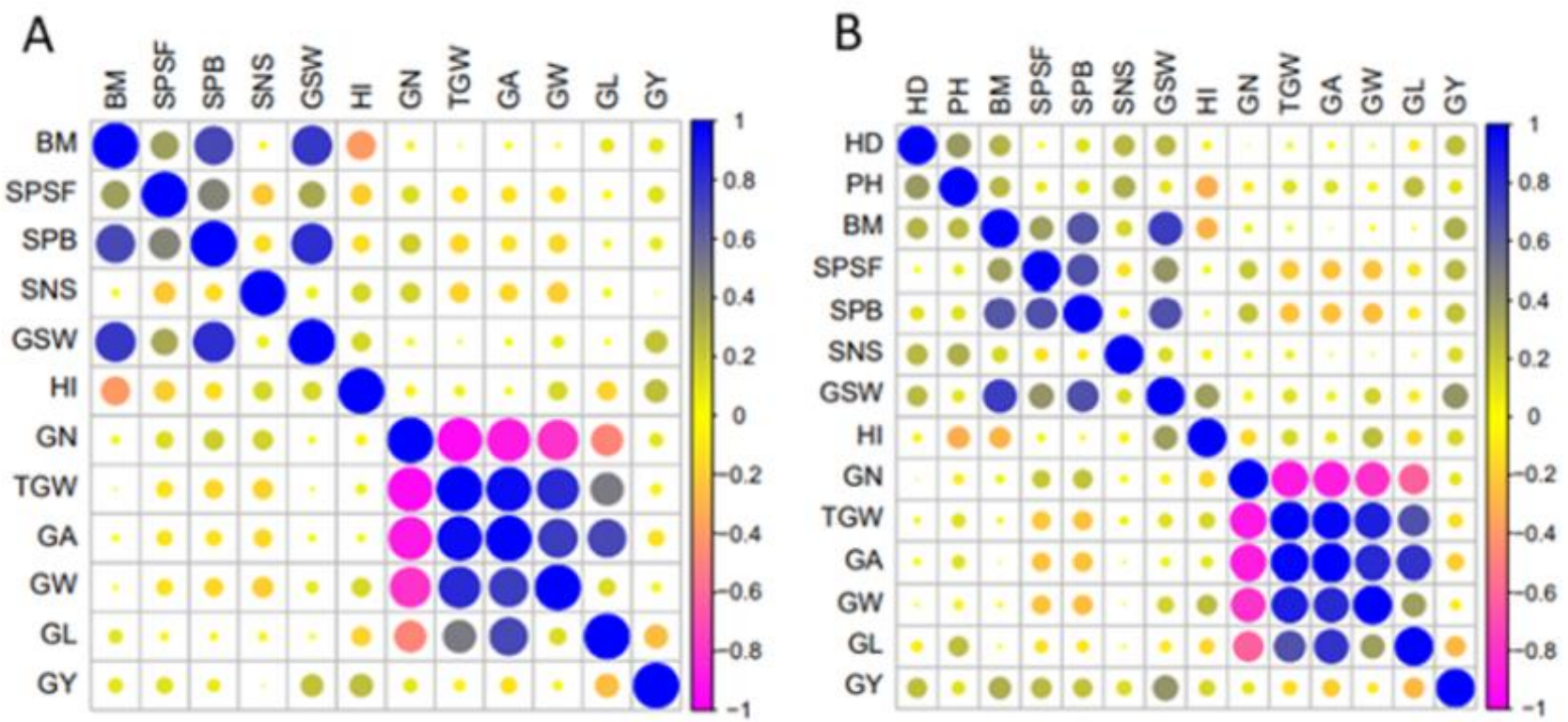

1005

Fig. 2 
1008
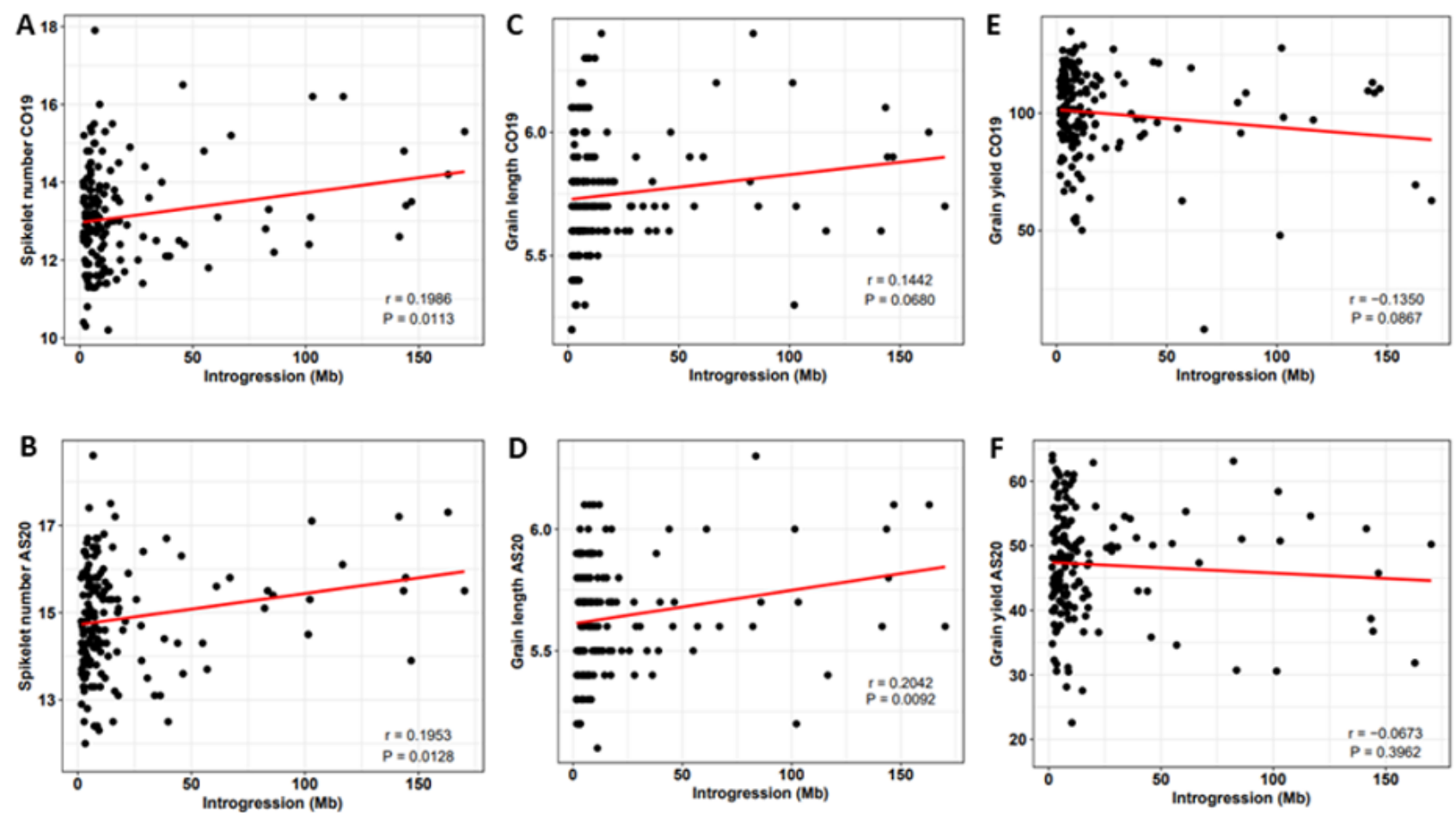

$1009 \quad$ Fig. 3

1010

1011
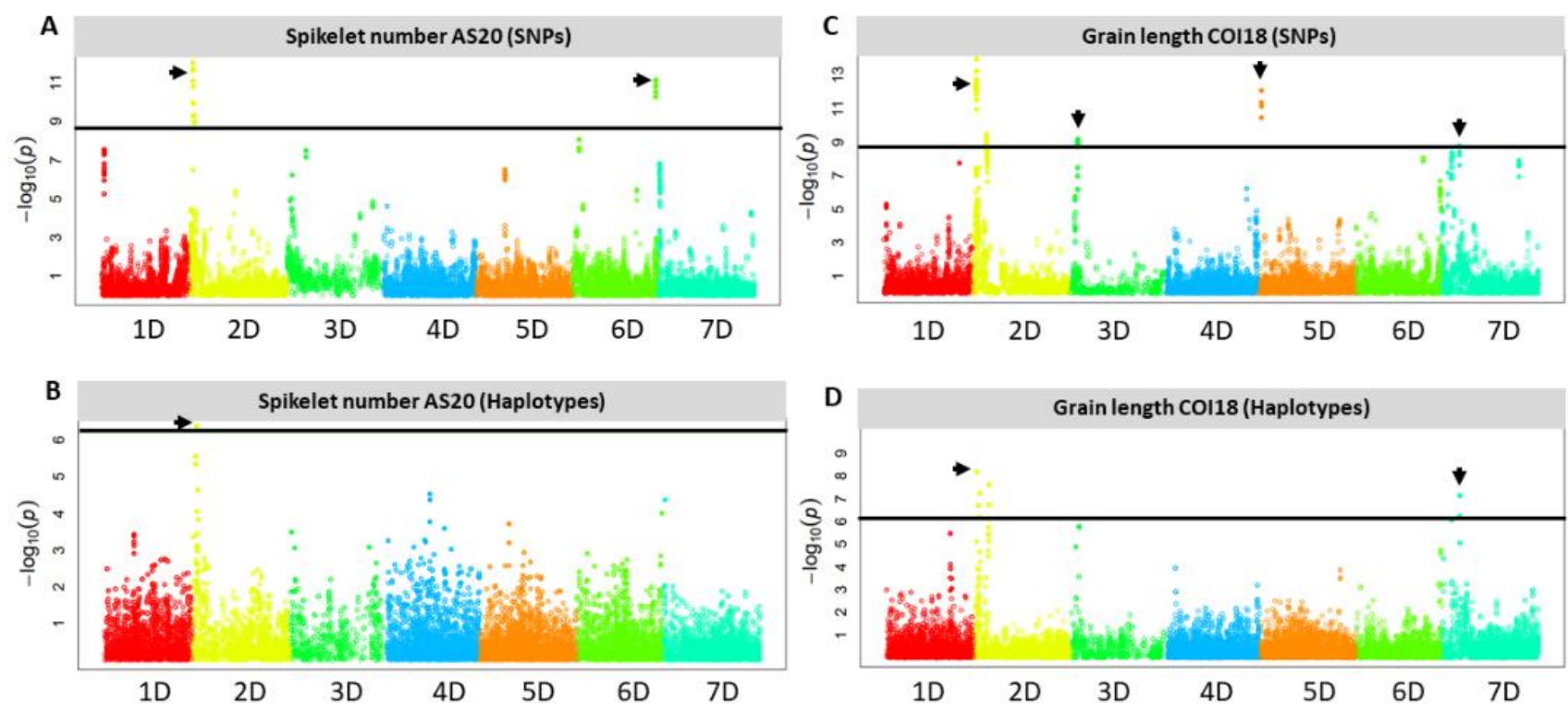

$1012 \quad$ Fig. 4

1013

1014

1015 
bioRxiv preprint doi: https://doi.org/10.1101/2021.05.29.446303; this version posted July 15, 2021. The copyright holder for this preprint (which was not certified by peer review) is the author/funder, who has granted bioRxiv a license to display the preprint in perpetuity. It is made available under aCC-BYGehletireatifectseafe.Ae. tauschii-wheat introgression

1016

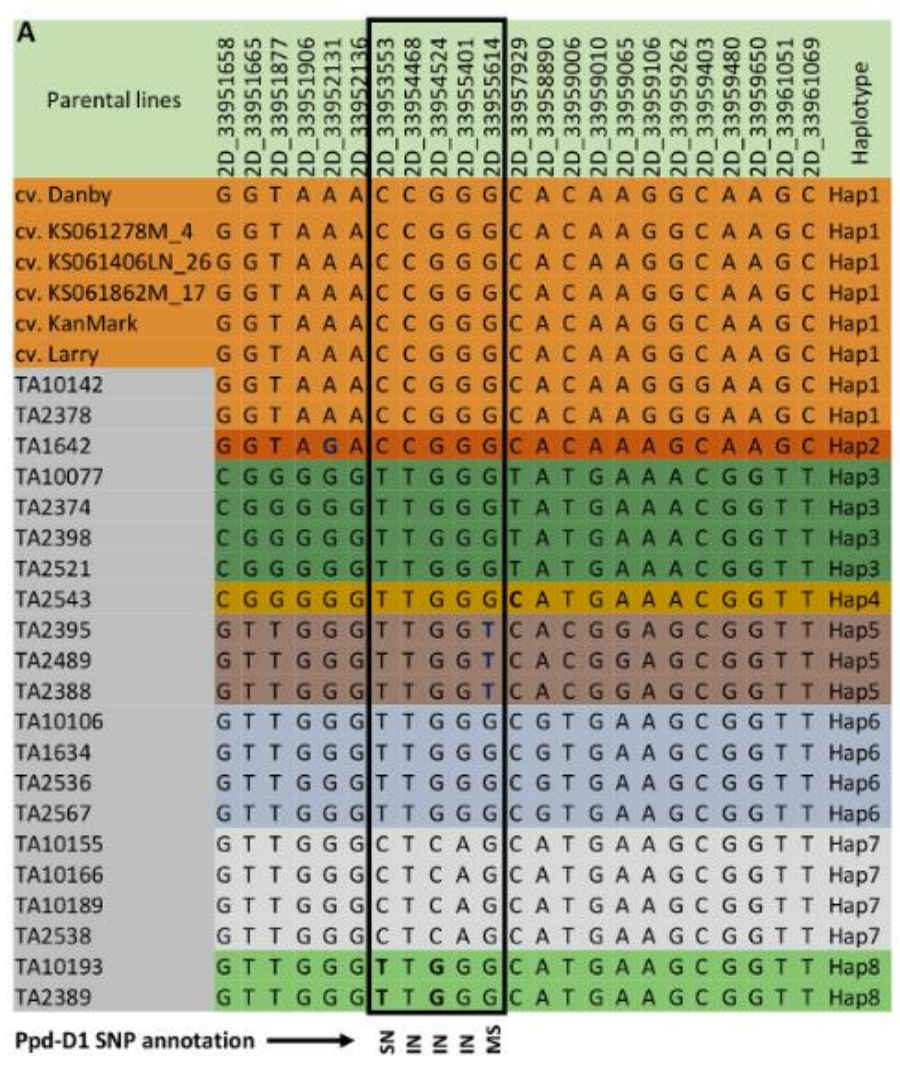

B

\begin{tabular}{|c|c|c|c|c|c|c|c|c|}
\hline \multirow[b]{2}{*}{$\begin{array}{l}\frac{0}{2} \\
\frac{0}{2} \\
\frac{0}{10}\end{array}$} & \multirow[b]{2}{*}{$\begin{array}{l}\text { むั } \\
\text { 壱 }\end{array}$} & \multicolumn{2}{|c|}{$\begin{array}{c}\text { SNS/HD } \\
\text { GWAS } \\
\text { SNPS }\end{array}$} & \multicolumn{2}{|c|}{$\begin{array}{l}\text { Ppd-D1 } \\
\text { locus tag } \\
\text { SNPs }\end{array}$} & \multirow[b]{2}{*}{$n$} & \multicolumn{2}{|c|}{ Mean \pm SE } \\
\hline & & 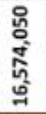 & 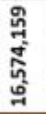 & 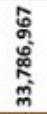 & 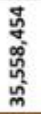 & & SNS & HD \\
\hline Hap_HW & - & $T$ & $T$ & A & A & 161 & $14 \pm 0.08$ & $200 \pm 0.16$ \\
\hline Hap_AeT* & + & C & A & G & A & 8 & $17 \pm 0.16$ & $202+0.91$ \\
\hline Hap_AeT & + & c & A & G & C & 10 & $15 \pm 0.32$ & $202 \pm 0.63$ \\
\hline
\end{tabular}
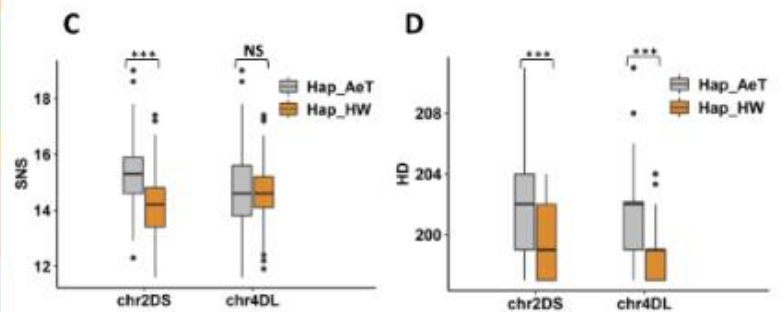

$\mathbf{E}$

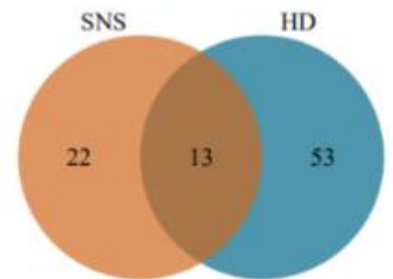

Fig. 5 